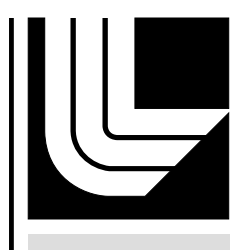

LAWRENCE LIVERMORE N A TIO NAL LABORATORY

Kshort Production in ALICE at the LHC

M. Tyler, R. Soltz

September 12, 2013 
This document was prepared as an account of work sponsored by an agency of the United States government. Neither the United States government nor Lawrence Livermore National Security, LLC, nor any of their employees makes any warranty, expressed or implied, or assumes any legal liability or responsibility for the accuracy, completeness, or usefulness of any information, apparatus, product, or process disclosed, or represents that its use would not infringe privately owned rights. Reference herein to any specific commercial product, process, or service by trade name, trademark, manufacturer, or otherwise does not necessarily constitute or imply its endorsement, recommendation, or favoring by the United States government or Lawrence Livermore National Security, LLC. The views and opinions of authors expressed herein do not necessarily state or reflect those of the United States government or Lawrence Livermore National Security, LLC, and shall not be used for advertising or product endorsement purposes.

This work performed under the auspices of the U.S. Department of Energy by Lawrence Livermore National Laboratory under Contract DE-AC52-07NA27344. 
CERN - "The spirit of CERN is scientific freedom and openness, which attracts the most extraordinary people." (Ginter, Heuer, \& Franzobel, p. 43)

The European Organization for Nuclear Research (CERN) was created in 1954 as international entity for the benefit of advancing the scope of knowledge in particle physics. The designation of CERN refers simply to an organization, which hosts the world's largest particle physics laboratory. CERN employs close to 2,400 full-time employees, 1,500 part-time employees and hosts 10,000 visiting engineers and scientists, affiliated with 608 universities and research facilities ranging from over 113 nationalities. (Wikipedia, CERN)

The European

Organization for Nuclear Research houses various colliders, which on a daily basis makes attempts at defining the initial anatomy of

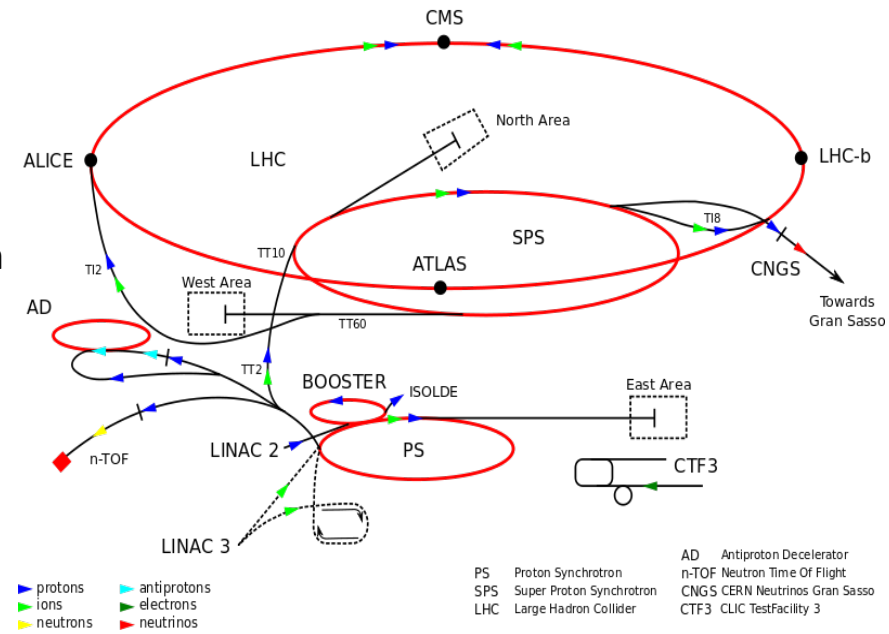
the universe. CERN utilizes the Figure 1 - Courtesy of Wikipedia world's largest and most sophisticated scientific tools, which aid in the analysis of clarifying the fundamental components of matter - Elementary Particles. These particles are forced into collisions that are near the speed of light or rather "relativistic". These collisions aid scientists and engineers who work at CERN in the endeavor of illustrating the process of how the fundamental forces of nature affect the interactions of particles/matter at various energy levels.

In the beginning there is always a theory (Ginter, Heuer, \& Franzobel, 2011, p. 45) 
CERN is composed of an enormous facility that spans about 150 square

kilometers in area (this includes a town-to-town radius encompassing all necessary administrative buildings, 6 accelerators, and 1 decelerator). The main site of CERN (Meyrin) is located at the outskirts of beautiful Geneva, Switzerland. CERN is duly noted historically for the inception of the World Wide Web and harnessing a large computer centre that facilitates powerful data processing, primarily for the benefit of experimental data analysis.

\section{The Large Hadron Collider}

The LHC is the world's largest collider to date. Known to most as the one ring to rule all, the LHC is composed of various different colliders adjoined together to reproduce a multiplicity of collisions at exceptionally high rates. The LHC pumps out a particular number particles and anti-particles in a certain manner of which are accelerated cyclically. Once these particles in the ring reach the required momentum, the ring then re-aligns in manner that guides these high velocity particles into one another thus propagating a Quark-Gluon Plasma phenomenon to occur. "The charged particles being accelerated are typically electrons, positrons, protons, or anti-protons, but depending on the application, they can also be ions in different states of charge, or even unstable isotopes." (Minty \& Zimmermann, 2003, p. 1)

The LHC has a 27 kilometer radius and is situated between Lake Geneva and French Jura. Most of the experiments at the LHC take place 100 meters underground (something similar to an Egyptian sepulcher but only 27 kilometers in radius).

In the beginning there is always a theory (Ginter, Heuer, \& Franzobel, 2011, p. 
CSU/LLNL/CERN Project 2013

R.A. Carmona | M.E. Tyler II

The term "Hadron" is a name associated with protons and nuclei, which both consist of quarks and can be accelerated in the LHC. A better analogy of what the collider achieves is paraphrased as "... particle beams with equivalent energy of 120 elephants traveling towards each other at $40 \mathrm{~km} / \mathrm{h}$ are smashed into each other." (Ginter, Heuer, \& Franzobel, 2011, p. 83)

These beams can be simplistically explained by CERN physicists as “... an ensemble of charged particles ( $a$ 'beam') is accelerated to high energy, and then is sent onto a fixed target or collided with another particle beam, usually of opposite charge and moving in the opposite direction." (Minty \& Zimmermann, 2003)

\section{ALICE - A Large lon Collision Experiment}

ALICE, one of 3 large experiments at the LHC, is composed of several elements. Starting from the interior to exterior, the barrel is composed of an Inner Tracking System (ITS) of six planes of high-resolution silicon pixel (SPD), drift (SDD), and strip (SSD) detectors, a cylindrical Time-Projection Chamber (TPC), three particle identification arrays of Time-of-Flight (TOF), Ring Imaging Cherenkov (HMPID) and Transition Radiation (TRD) detectors, and two electromagnetic calorimeters (PHOS and EMCal).

Tracking detectors:

Inner Tracking System (ITS) (Carminati, et al., 2004)

$\rightarrow$ the basic functions of the inner tracker are:

In the beginning there is always a theory (Ginter, Heuer, \& Franzobel, 2011, p. 
- secondary vertex reconstruction of heavy flavour and strange particle decays,

○ particle identification and tracking of low-momentum particles, and

- improvement of the impact parameter and momentum resolution.

- The structure of the ITS is a 6 layer, cylindrical silicon vertex detector, (CERN, 2008, pp. 6-8)

\section{Time Projection Chamber (TPC)}

Allows for precise position and linear energy loss measurements with a gas gain of the order of $10^{4}$. With a resolution to this degree the TPC can serve, in addition to tracking, as a detector for particle identification in the region of the relativistic rise, up to momenta of order $50 \mathrm{GeV} / c$. The designs of the readout chambers and electronics, as well as the choice of the operating gas, are optimized for good double-track resolution and minimal space charge induced distortions.

\section{Transition Radiation Detector (TRD)}

Utilized for tracking electrons in the central region improving the transverse momentum $\left(\mathrm{p}_{\mathrm{T}}\right)$ resolution at high momentum (those magnitudes above $1 \mathrm{Giga-eV/c).}$ Below this momentum electrons can be identified by the specific energy loss measurement in the TPC. Above $1 \mathrm{GeV} / \mathrm{c}$ transition radiation (TR) from electrons passing a radiator can be exploited in concert with specific energy loss in a suitable gas mixture to obtain the necessary pion rejection capability Essentially, the TRD was designed to derive a fast trigger for charged particles with high momentum. (CERN, 2008, p. 66)

In the beginning there is always a theory (Ginter, Heuer, \& Franzobel, 2011, p. 4 45) 
The TRD's overall design parameters are the following:

- Pion rejection capability

- Position and momentum

- Radiation Length

- Detector granularity

\section{Time-of-Flight (TOF)}

The TOF detector is a large area array that covers the central pseudo-rapidity region for Particle Identification (PID) in the intermediate momentum range. The TOF, coupled with the ITS and TPC for track and vertex reconstruction and for change of energy with respect to time $(\mathrm{x})$ measurements in the low-momentum range, this provides event-by-event identification of large samples of pions, kaons, and protons see invariant mass studies. (CERN, 2008, p. 74)

\section{High Momentum Particle Identifier (HMPID)}

The HMPID detector is designed to capture inclusive measurements of identifiable hadrons at transvers momentums greater than 1 Giga-Electron Volt per unit of speed of light. The goal is to refine the particle identification capabilities of ALICE via enabling identification of charged hadrons beyond the momentum interval attainable through energy-loss (within the TPC and ITS) and TOF measurements. Also, identification of light nuclei and anti-nuclei at high transverse momenta in the central rapidity region can be detected. (CERN, 2008, p. 83)

\section{Photo-Spectrometer (PHOS)}

In the beginning there is always a theory (Ginter, Heuer, \& Franzobel, 2011, p. 5 45) 
The PHOton Spectrometer is a high-resolution electromagnetic spectrometer covering a limited acceptance domain at central rapidity. The main goals of this detector is test the thermal and dynamical properties of the initial phase of the collision extracted from low transverse momentum direct photon measurements and the study of jet quenching through the measurement of high-transverse momentum pion( 0$)$ and gamma-jet correlations. (CERN, 2008, p. 87)

\section{Electromagnetic Calorimeter (EMCal)}

An Electromagnetic Calorimeter is composed of a lead-scintillator sampling calorimeter integrated with longitudinal wavelength-shifting fibers, producing a read out via avalanche photo diodes. The EMCal is optimized to measure jet production rates and fragmentation functions in conjunction with the charged particle tracking in the other barrel detectors. (CERN, 2008, p. 22)

\section{Muon Spectrometer (Essential to PRL - (Abelev, et al., 2012))}

This detector is placed outside of the L3 Magnet where most of the well-noted detectors are seated. Other smaller or hard to notice detectors are the: ZDC, PMD, FMD, T0, and V0. These detectors are noted for having a 'fast trigger capability' (CERN, 2008, p. 8).

The muon Spectrometer is performed in the pseudorapidity region. The muon Spectrometer allows for measurement of a complete spectrum of heavyquark vector-mesons resonances in a muon plus to

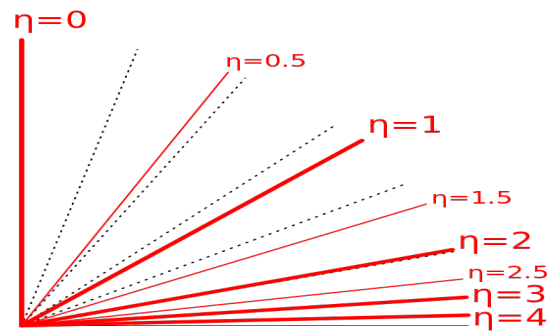

Figure 2 - Pseudo-rapidity courtesy of Wikipedia

muon negative channel. In terms of high-luminosity

In the beginning there is always a theory (Ginter, Heuer, \& Franzobel, 2011, p. 45) 
runs, the muon spectrometer will gather data collectively from a limited number of ALICE detectors (such as ZDC, SPD, PMD, T0, V0, and FMD). (CERN, 2008, p. 105)

\section{Zero Degree Calorimeter (ZDC)}

The ZDC aids in estimation of Centrality for a participating nucleons. The ZDC accomplishes this via measuring the energy carried in the forward direction (at 0

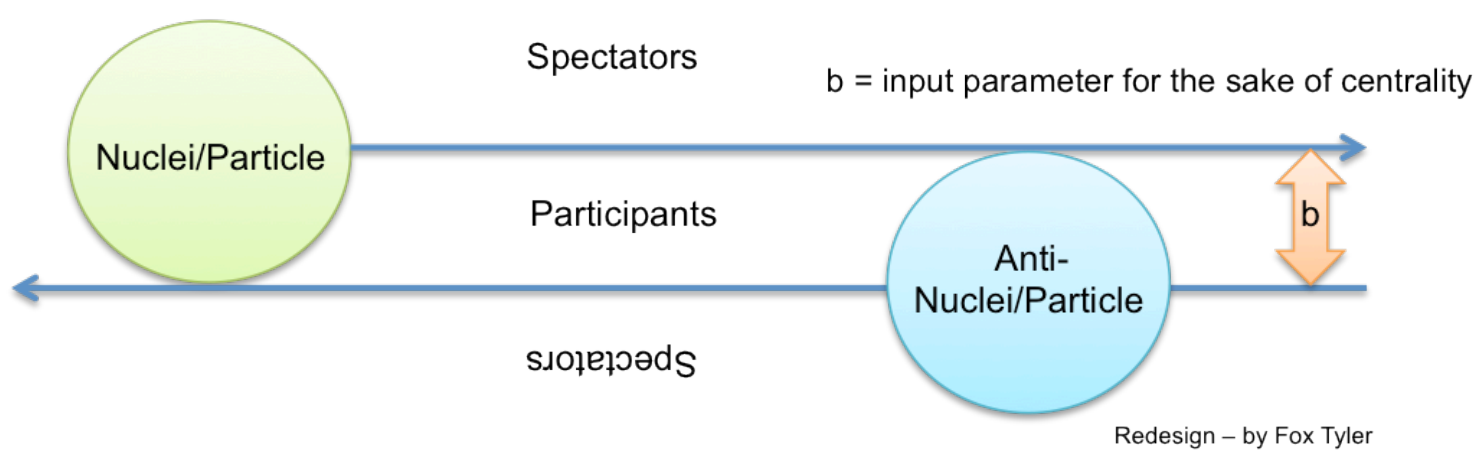

Figure 3 - 'Centrality' determination via ZDC

degrees relative to the beam direction) by non-interacting (spectator) nucleons. If all the spectators are detected, the number of participants can be properly estimated. The centrality information provided by the ZDC is also used for triggering at Level (L1). Lastly, the ZDC is also a positron-sensitive detector, which can give an estimate of the reaction plan in nuclear collisions. (CERN, 2008, p. 115)

\section{Photon Multiplicity Detector (PMD)}

The PMD measures the multiplicity and spatial distribution of photons in the forward pseudo-rapidity region. Such measurements yield estimations for transverse electromagnetic energy and the reaction plane on an event-by-event basis. Quantifying the amount of photon multiplicity provides scientist with significant information in 
CSU/LLNL/CERN Project 2013

R.A. Carmona | M.E. Tyler II

terms of limiting fragmentation, order of phase transition, the equation of state of

matter and the formation of disoriented chiral condensates. (CERN, 2008, p. 120)

\section{Forward Multiplicity Detector (FMD)}

The essential function of the FMD is to provide charged-particle multiplicity parameters for a particular pseudo-range. These parameters can be combined with the pseudo-rapidity coverage parameters of the ITS pixel detector which monitors the nominal vertex position. These parameters (those from the FMD and the ITS pixel detector) are overlapped in order to provide redundancy and crosscheck measurements between sub-detectors and guarantee that continuous coverage for a distribution of vertices along the z-axis. (CERN, 2008, p. 125)

\section{TO Detector (Essential Initial Conditions Detector)}

The T0 detector was created to handle the following objectives: (1) Generation of a start time (TO) for the TOF detector. Such a timing signal is required in order to ascertain the real time of collision (plus a fixed time delay) and is independent of the position of the vertex. The necessary precision of such a signal is about 50 ps (r.m.s.). (2) Next, the T0 detector has to measure the vertex position (with a precision of plus or minus $1.5 \mathrm{~cm}$ ) for each interaction and to provide a L0 trigger when the position is within the preset values. This will aid in filtering out beam-gas interactions. The T0 detector can also generate an early 'wake-up' signal to the TRD detector, prior to the L0 trigger. Also, the TO detector provides redundancy to the VO counters and can generate minimum bias (one or both arrays hit) and multiplicity trigger (semi-central and central). (CERN, 2008, p. 136)

In the beginning there is always a theory (Ginter, Heuer, \& Franzobel, 2011, p. 45) 
V0 detector (Essential to PRL - (Abelev, et al., 2012))

The $\mathrm{V} 0$ detector has a variety of functions. It provides minimum-bias triggers for the central barrel detectors in $\mathrm{pp}$ and A-A collisions. These triggers are given by particles originating from initial collisions and from secondary interactions in the vacuum chamber elements. The V0 detector essentially serves as an indicator of the centrality of the collision via the multiplicity recorded in the event. Finally, the V0 detector aids in the quantification of Luminosity in pp collisions with a decent precision of about $10 \%$. (CERN, 2008, p. 132) 
1 What is strangeness and how it fits in heavy - ion Research?

"In our modern understanding, strangeness is conserved during the strong and the electromagnetic interactions, but not during the weak interactions." (Wikipedia, Strangeness, 2010)

In the initial stage of the Quark-Gluon Plasma creation, the ' $u$ ' and 'd' quarks are broken apart from the participating yet opposing parent particles after the fireball-like collision. A high momentum collision is required for this phenomenon to occur. Such a collision generates a fireball that is approximately 100,000 times the core temperature of the sun. Since, such an exorbitant amount of heat is generated at the inception - an exact measure of the QGP is not possible. Instead, through the use of ALICE's various sophisticated detectors a recreation of the data from jet steams is our reference point within heavy-ion research in ascertaining an understanding of what the ' $s$ ' quark is about. The ' $s$ ' quark is heavier than the ' $u$ ' or 'd' quarks, yet comparable in mass enough to undergo production and modification processes in a like fashion. (Abelev B. , 2012)

Strangeness is measures as such below:

$$
S=-\left(n_{s}-n_{\bar{s}}\right)
$$

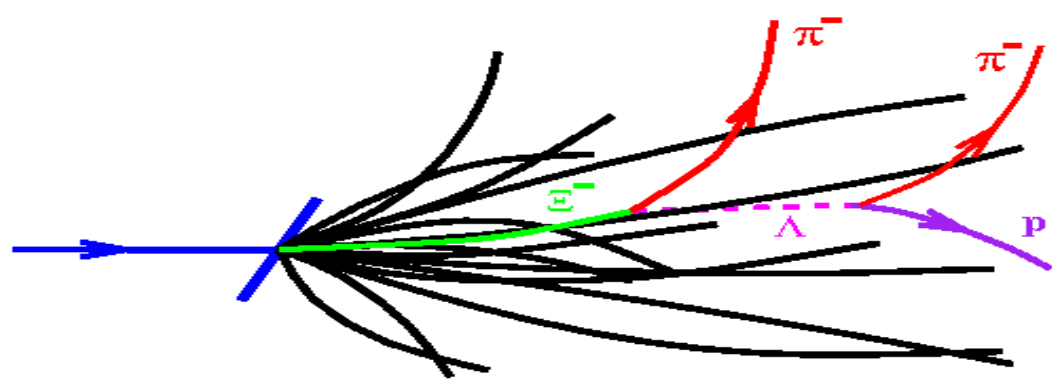

Figure 4 - Strangeness production [9]

2. What will you be measuring/analyzing

By examining the decayed jet stream of escaped daughter particles (Kaon-shorts and Lambdas-Zeros to name a few), we will be able to reverse investigate these remnants of parent particles in order to

In the beginning there is always a theory (Ginter, Heuer, \& Franzobel, 2011, p. 10 45) 
conceptualize the original quarks during the QGP phase and attempt to understand strangeness at it beginnings. We accomplish this by analyzing the Z-Vertex and Invariant Mass distributions as supplied via Monte Carlo simulations downloaded from the ALICE repository.

Invariant Mass Calculations - a running thesis

So, imagine two mosquitoes colliding into each other - the required energy necessary for this event to occur is about 1.2 micro-Joules. Well, after that collision has occurred various components of fragmented matter would scatter into various directions (hence the fireball-to-QGP transition).

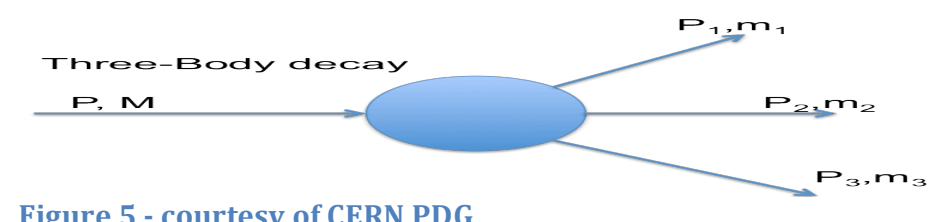

Figure 5 - courtesy of CERN PDG

For the sake of comprehension, lets regulate this phenomena to a 3-momentum component ' $P$ ' of mass and a necessary component of Energy ' $E$ '. Viewing the event in this fashion aids us in grasping the purpose and need of a Lorentz Transformation.

Let's think through this a bit. When done in this fashion, we can express Einstein's equation for the energy of an object:

$$
E=M C^{2}
$$

into a more relativistic version and then begin to full grasp the purpose of Invariant Mass (also known as the rest mass, $m$ ) as related to high energy $p$-p or Au-Au collisions.

$$
E=m \gamma C^{2}
$$

(where ' $\gamma$ ' the Lorentz Gamma Factor)

$$
\gamma=1 / \sqrt{\left(1-\frac{v^{2}}{c^{2}}\right)}
$$

Invariant mass is considered as the rest mass of which has characteristics of the total energy and momentum of a system. Now, if this system is comparable to simulations generated in Monte Carlo via Alice we have:

$$
m=E /\left(\gamma * c^{2}\right)
$$

In the beginning there is always a theory (Ginter, Heuer, \& Franzobel, 2011, p. 


$$
m=\frac{E}{c^{2}}\left(\sqrt{\left(1-\frac{v^{2}}{c^{2}}\right)}\right)^{(-1)}
$$

This rest mass is the intrinsic data that physicists utilize in order to carefully track various new particles created from the Quark-Gluon Plasma expansion - similar to the big bang in essence.

Say for instance a lambda particle is being observed in terms of Invariant Mass:

$$
\begin{gathered}
\Lambda=\text { Mass }=f\left(\bar{P}_{1}, \bar{P}_{2}\right) \\
\Lambda=\text { Mass }=f\left(\left(\overrightarrow{P_{1}, E_{1}}\right),\left(\overrightarrow{P_{2}, E_{2}}\right)\right)
\end{gathered}
$$

where:

$$
\begin{aligned}
& P_{1}=P_{x 1}, P_{y 1}, P_{z 1}, E_{1} \\
& P_{2}=P_{x 2}, P_{y 2}, P_{z 2}, E_{2}
\end{aligned}
$$

say $S=$ Invariant Mass, then we have:

$$
\left.S=\sum \overrightarrow{(P}^{2}-\vec{E}^{2}\right)
$$

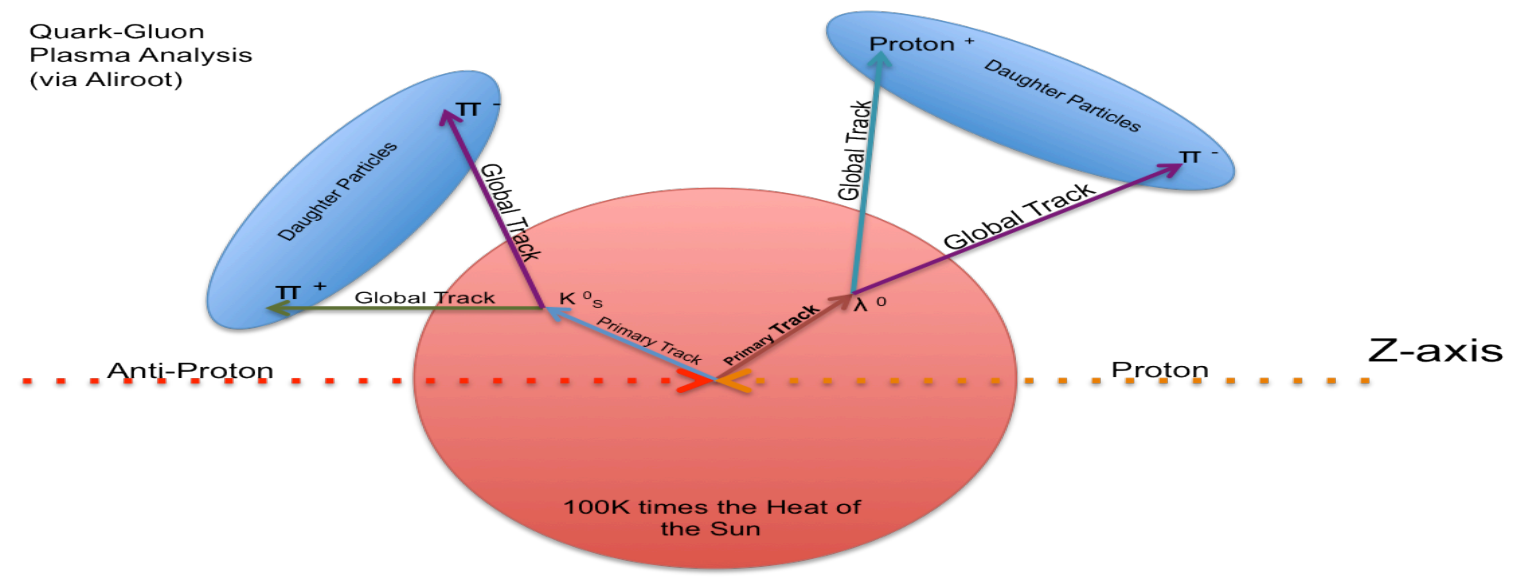

Figure 6 - Overview of Analysis

3. What do you expect to get from your research?

A simplistic breakdown of what nuclear physicist do and how this can be an inspiring experience for all ages. Learning for the sake of

In the beginning there is always a theory (Ginter, Heuer, \& Franzobel, 2011, p. 
knowledge is in my opinion transformative teaching when performed from the guise of innovation. As the old saying has always gone, "in order to teach someone about a drop of water, you would have to know about a gallon." In like manner, studying the physics involved with ALICE will aid me teaching Physics to high-needs secondary schools in the Chicago-land area.

When most people think of CERN or even nuclear physics, the response is "oh my god" and they attempt to run away. I believe there is a better way to introduce science of this caliber in a manner that not only inspires students but also prepares them from the next wave of future STEM professions. At the end of this CSU/LLNL/CERN journey, I will have accomplished this by forcing myself to understand the complex caveats of Aliroot (which is $\mathrm{C}++$ training basically - all over again) and through this experience will know how to teach programming to students if need be. Also, I expect that I will be able connect students with the most patient and knowledge teachers in the field of science in order that they may flourish for as long as their hearts desire.

In the beginning there is always a theory (Ginter, Heuer, \& Franzobel, 2011, p. 
Part 3 | Methodology

AliRoot had an initial start in 1998 within the ALICE software environment. This implementation was at a time when computing in high-energy physics was facing a daunting objective. A specific network of a thousand developers and users had to be quickly converted from a procedural language such as (FORTRAN) which was utilized for 40 years prior into a comparatively new language $(\mathrm{C}++)$. Another issue arose from this conversion process at this time. The ALICE had to be transitioned from being

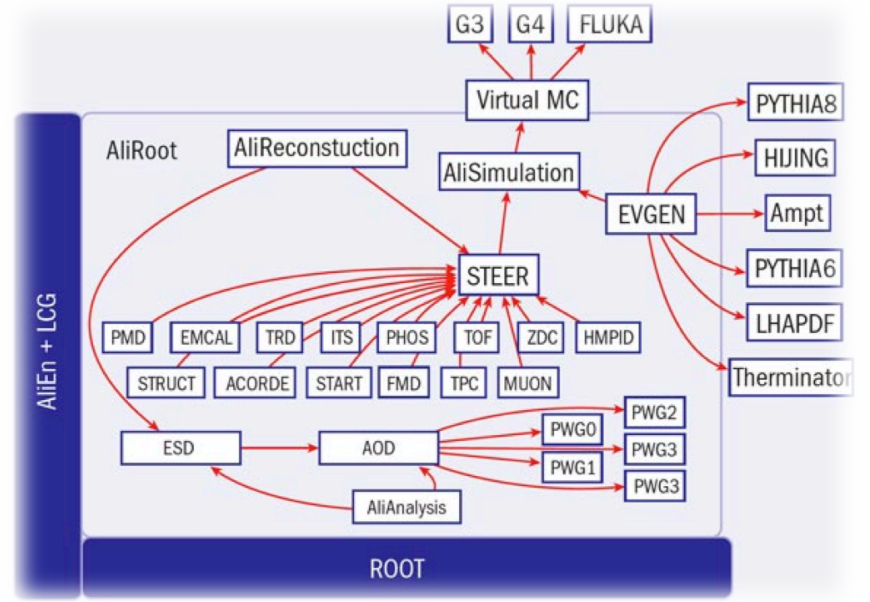

Figure 7 - Schematic representation of ALiRoot courtesy of CERN Courier 2012

composed of loosely connected

computer nodes to an effectively connected Grid infrastructure. In order to make this endeavor (Aliroot) a reality, a detector simulation package coupled into a C++ "class" combined with ROOT (the $\mathrm{C}++$ framework of which provided the data manipulation and analysis constructs that Rene Brun and his team developed for the LHC experiments) was required (Carminati F. , 2012).

The type of research that the CSU ALICE team will be conducting is in the category of "Offline data processing" and is 'Chaotic' (as opposed to 'Cathedral') in nature. This includes the typical offline activities of calibration, alignment, reconstruction, simulation and analysis, both centrally organized and end-user driven. The offline data processing typically uses the framework for distributed computing and only some rare cases runs on local resources (in our case - PC or Mac based laptops). (CERN, 2008, pp. 162-163) 
Since this is an offline track reconstruction, the 'coaches' (algorithms) are based on a Kalman filter approach. ROOT tasks are handled via an "analysis train", where essentially one event is read in memory and then passed to the different analysis tasks (this sequence is analogous to the manner is which wagons are linked to a train for transport).

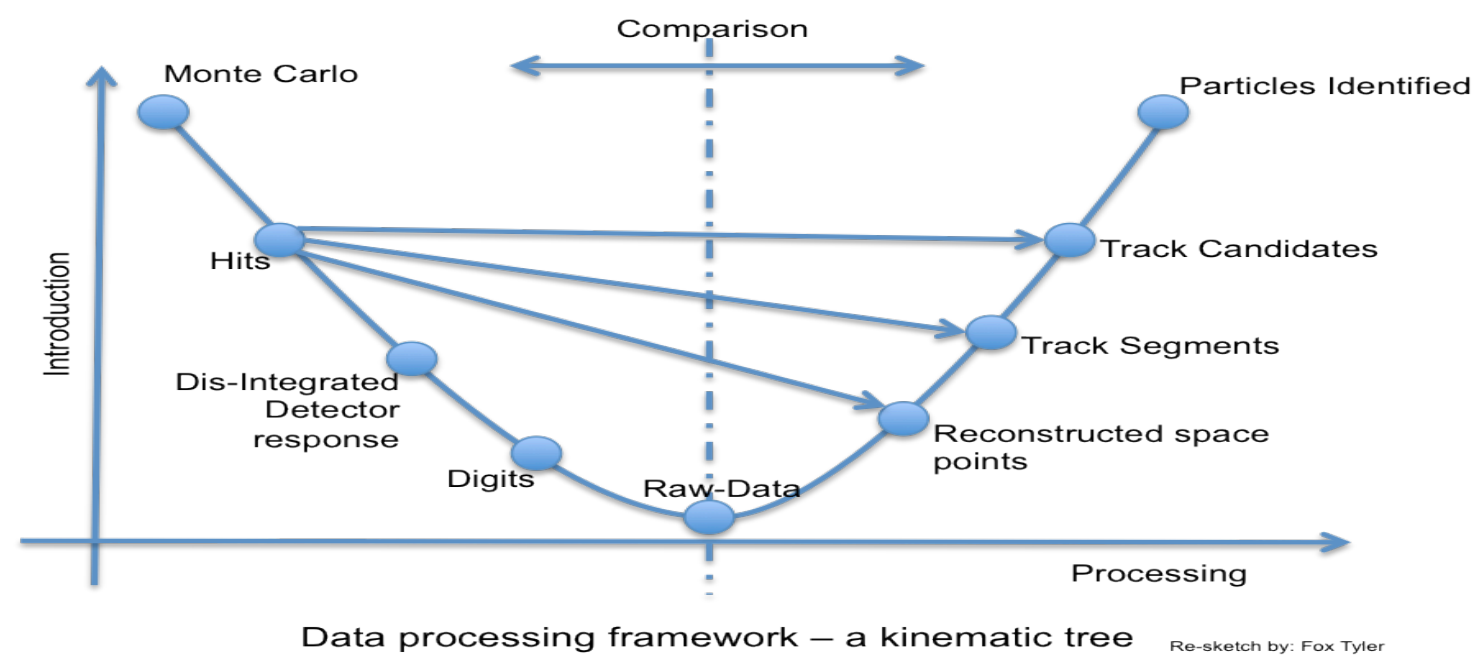

Figure 8 -Histogram reproductions courtesy of CERN 2008

The role of the AliRoot framework is illustrated above. This kinematic tree, for example, illustrates the physics processes at the parton level and the results of the fragmentation (primary particles), which are created by the event generators. The data provided by the event generators contain full information about the generated particles: type, momentum, charge, and mother-daughter relationship. During transport, the response of the detectors to each traveling particle is simulated. The hits (energy deposition at a particular point and time) are gathered from each detector. This information is accompanied by the 'track references' corresponding to the location where the particles have traveled based upon end-

In the beginning there is always a theory (Ginter, Heuer, \& Franzobel, 2011, p. 15 45) 
user defined coordinates (reference planes). These hits are converted into digits,

which take into account each particular detector and the associated response signal from the hardware itself. From this point, the reconstruction chain/process is initiated. (CERN, 2008, p. 171)

$\underline{\text { Final Analysis }}$

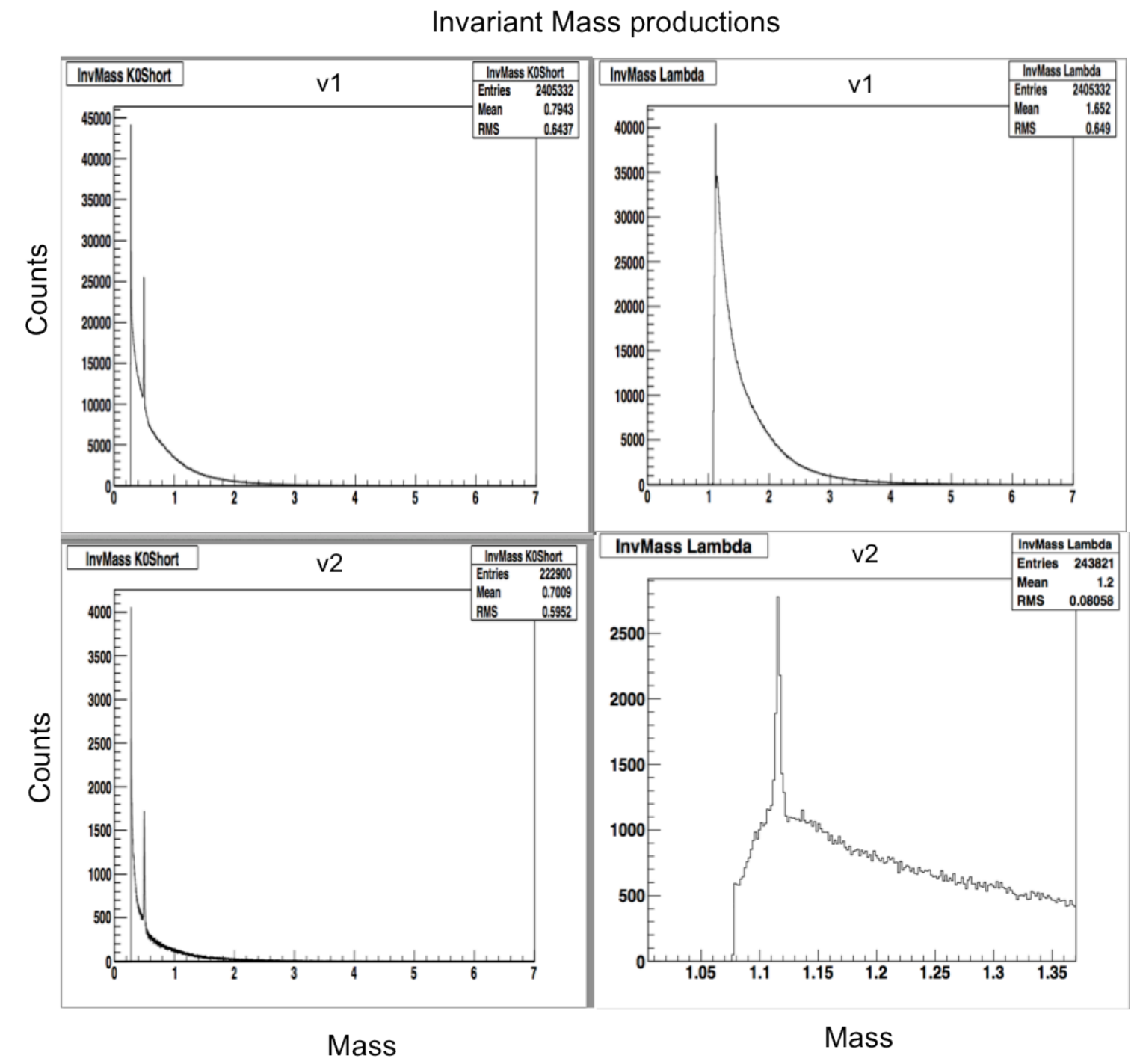

Figure 9 - Overall Invariant Mass distributions via Aliroot

As shown above in figure 9, analysis of tracks as collected from the data repository are sampled and prepared by utilizing AliRoot macros. In this figure

In the beginning there is always a theory (Ginter, Heuer, \& Franzobel, 2011, p. 16 45) 
Invariant Mass histograms are retrieved, cut, and superimposed to provide an initial efficiency histogram for further analysis (see figure 13). This process is sequentially accomplished by distinguishing the difference between invariant mass peaks (as referenced via the P.D.G. database for particular particles) and noise/interference signatures included with the sample (see figure 11). Within AliRoot, we programmatically targeted noise/interference signatures and made specific 'cuts' to remove high magnitudes of irrelevant data values. Following this procedure, a re-fit or progression alignment curve (see figure 12) of the altered histogram was processed and an overlay of the resulting histogram was superimposed together with the raw data histogram lastly.

The research focus was primarily centered on Kaon (Short Life) and Lambda 'Strange' particles. For illustrative purposes, the Kaon (Short Life) will mainly be discussed in this paper.

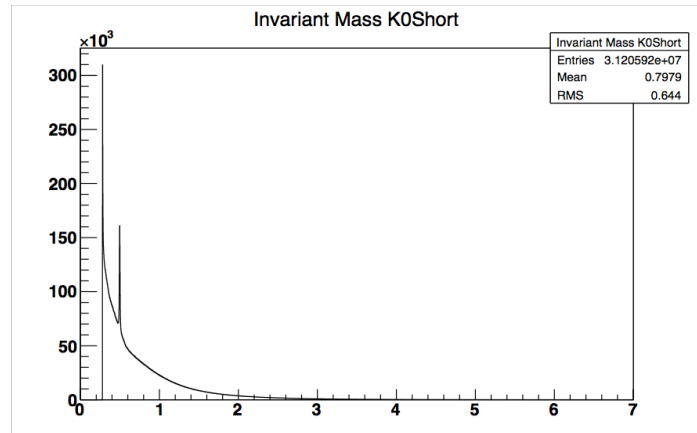

Figure 10 - K-Short Raw Data

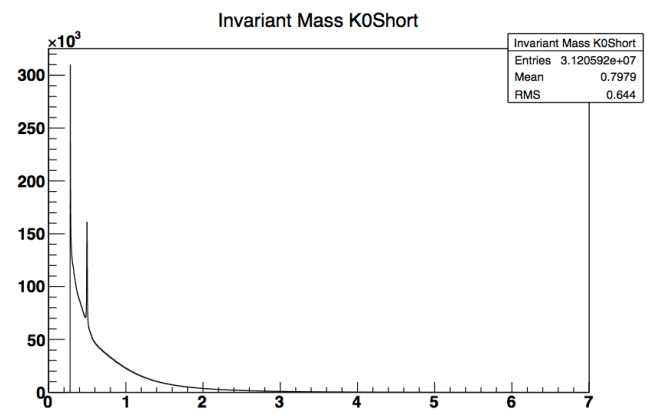

Figure 11 - K-Short Background/Noise

In the beginning there is always a theory (Ginter, Heuer, \& Franzobel, 2011, p. 


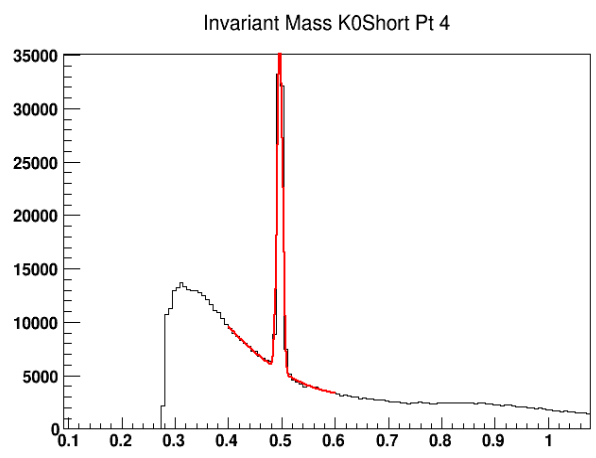

Figure 12 - K-Short (Raw Data w/ modified Background after cuts)

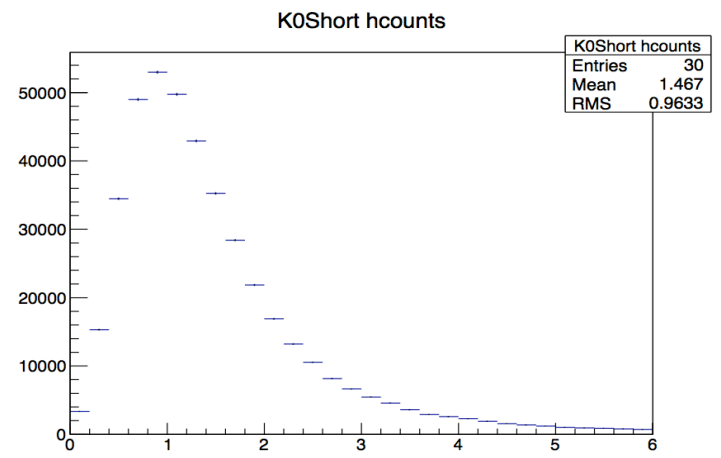

Figure 13 - K-Short Overall efficiency histogram for various cuts

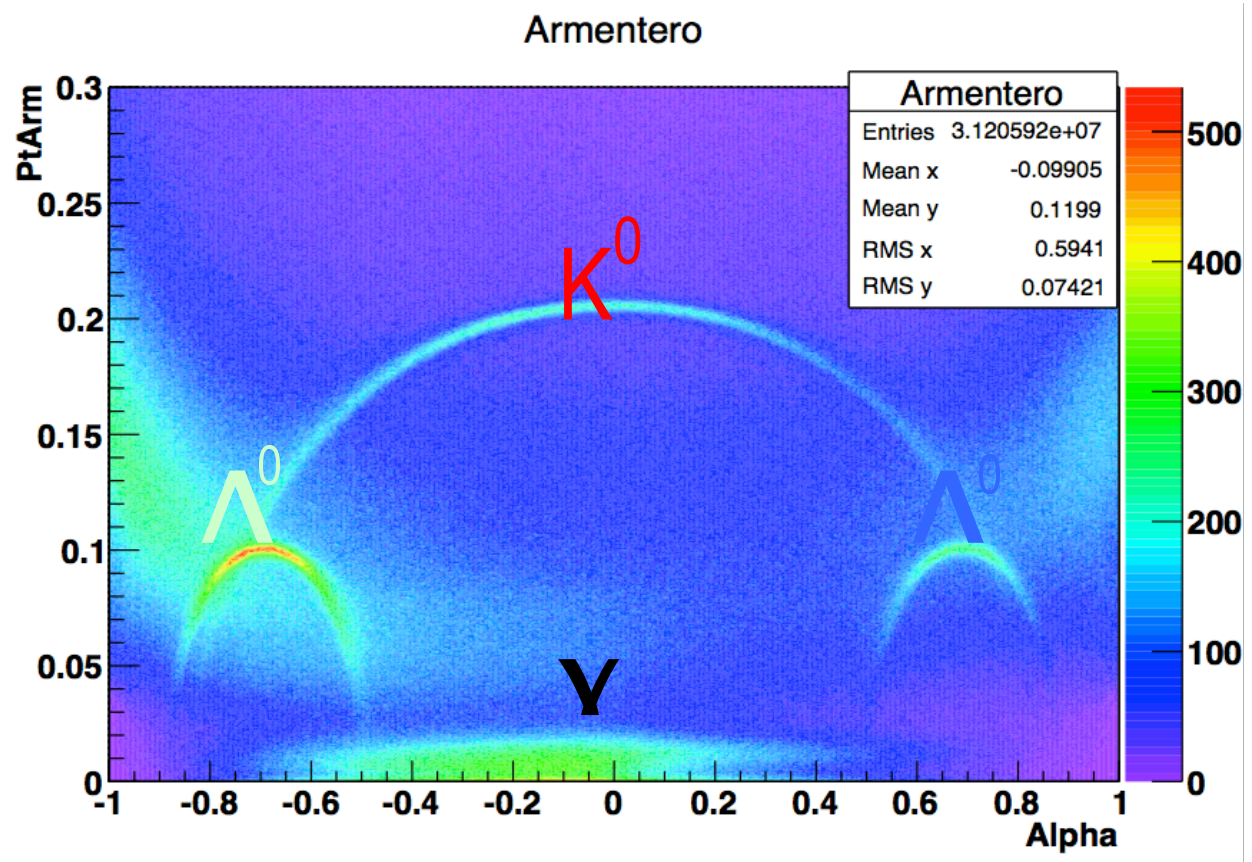

Figure 14 - Armentero distribution (Quality check of the V0)

This sample, cut, progression fit and superimpose strategy was utilized for several data points for both types of 'Strange' particles. The resulting identification plots (Armenteros plot is shown in figure 14) where is possible to clearly distinguish the lines for the K-short, lambda and antilambda.

In the beginning there is always a theory (Ginter, Heuer, \& Franzobel, 2011, p. 


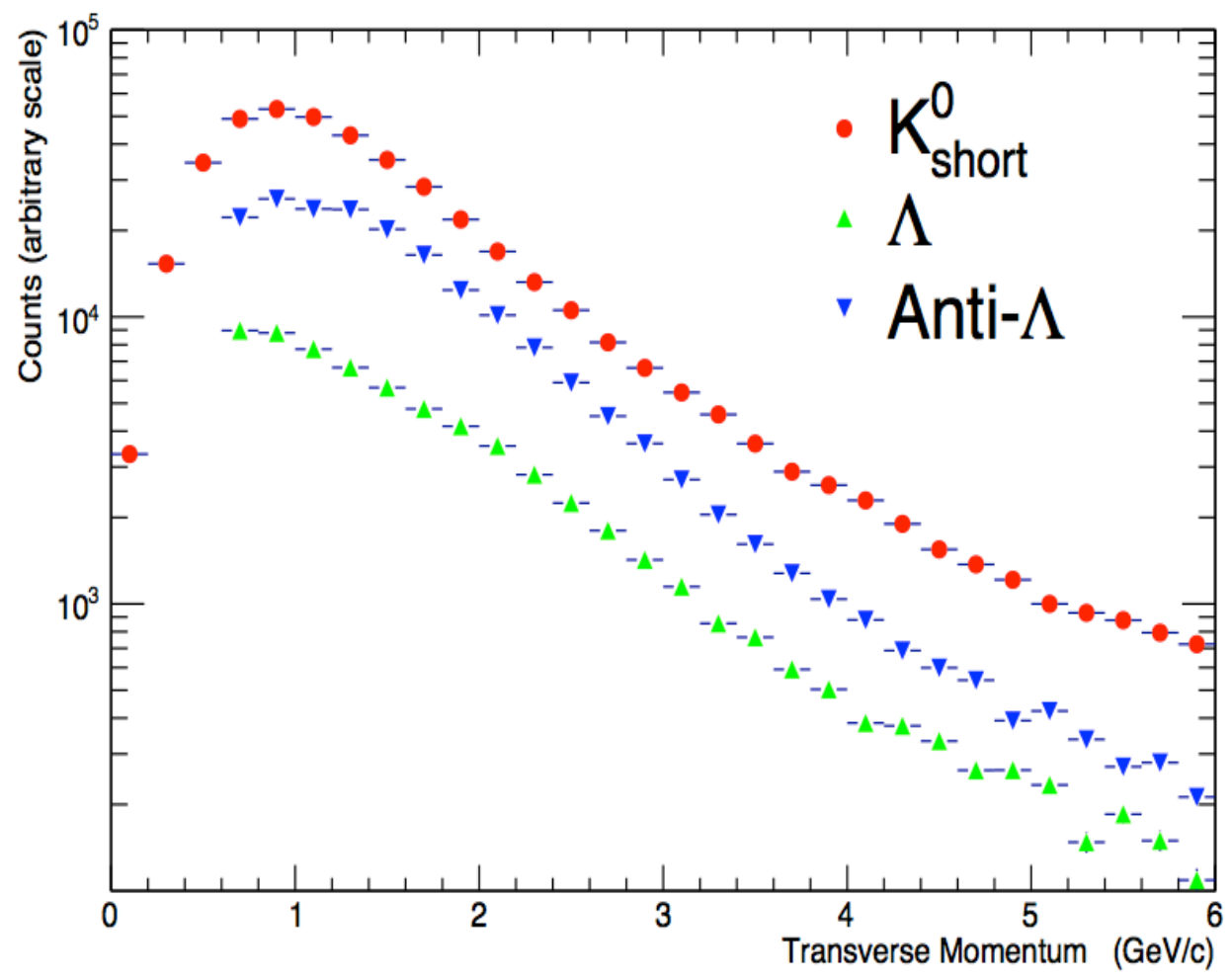

Figure 15 - Final transverse momentum distribution histogram for K-short and lambdas

This procedure was also performed for the anti-lambda data samples and overall (lambda, Kaon, and anti-lambda) transverse momentum histogram was produced (see figure 15).

In the beginning there is always a theory (Ginter, Heuer, \& Franzobel, 2011, p. 
The next step in this work will be to calculate the efficiencies to correct the momentum distribution spectra of the strange particles using Monte Carlo simulations.

\section{Works Cited}

Aamodt, K., Abelev, B., Quintana, A., Adamova, D., Adare, A., Aggarwal, M., et al. (2010). Charged-Particle Multiplicity Density at Midrapidity in Central Pb-Pb Collisions at sqrt(Snn) $=2.76 \mathrm{TeV}$. Physical Review Letters, 105, 1-11. Aamodt, K., Quintanna, A. A., Adamova, D., Adare, A. M., Aggarwal, M. M., Rinella, G. A., et al. (2011). Strange particle production in proton-proton collisions at $\operatorname{sqrt}(\mathrm{s})=$ $0.9 \mathrm{TeV}$ with ALICE at the LHC. The European Physical Journal C , 1-24.

Abelev, B. (2012, 6 22). Strangeness at the new energy frontier, from pp to PbPb. (A. M.-A. Experiment, Producer, \& European Organization for Nuclear Research) Retrieved 6 26, 2013, from ALICE Matters: Strangeness at the new energy frontier, from $\mathrm{pp}$ to $\mathrm{PbPb}$

Abelev, B., Adam, J., Adamova, D., A., M. A., Aggarwal, M. M., Rinella, G. A., et al. (2012). $\mathrm{J} / \mathrm{Psi}$ Suppression at Forward Rapidity in Pb-Pb Collisions at sqrt(Snn) $=$ 2.76 TeV. Physical Review Letters, 109.

Carminati, F. (2012, March 27). The ALICE computing project - Resume. Retrieved 71 , 2013, from CERN Courier: http://cerncourier.com/cws/article/cern/49059

Carminati, F., Foka, P., Giubellino , P., Morsch, A., Paic, G., Revol, J.-P., et al. (2004). ALICE: Physics Performance Report .JOURNAL OF PHYSICS G: NUCLEAR AND PARTICLE PHYSICS , 1, 1517-1749.

CERN, 2. J. (2008). The ALICE experiment at the CERN LHC. The CERN Large Hadron Collider: Accelerator and Experiments , 245.

Collaboration, T. A. (2010). Strange particle production in proton-proton collisions at sqrt(s) $=0.9 \mathrm{TeV}$ with ALICE at the LHC. Eurpoean Organization for Nuclear Research .

Ginter, P., Heuer, R.-D., \& Franzobel. (2011). LHC - Large Hadron Collider. Genva, Switzerland, United Nations : UNESCO Publishing .

Minty, M., \& Zimmermann, F. (2003). Measurement and Control of Charged Particle Beams. Berlin, Germany: Springer.

Salur, S. (2006). Investigation of Hadronic Resonances with STAR. Yale University Dissertation for Candidacy for the Degree of Ph.D , 184.

Wikipedia. (n.d.). CERN. Retrieved 6 21, 2013, from Wikipedia: https://en.wikipedia.org/wiki/CERN Wikipedia. (2010, 7 10). Strangness . Retrieved 6 25, 2013, from Wikipedia: http://en.wikipedia.org/wiki/Strangeness

In the beginning there is always a theory (Ginter, Heuer, \& Franzobel, 2011, p. 20 45) 